Copyright by the American Physical Society. Holtz, M.; Sauncy, T.; Dallas, T.; Massie, S.,

"Effect of pressure on defect-related emission in heavily silicon-doped GaAs," Phys. Rev. B 50, $14706(\mathrm{R})$ DOI: http://dx.doi.org/10.1103/PhysRevB.50.14706

\title{
Effect of pressure on defect-related emission in heavily silicon-doped GaAs
}

\author{
M. Holtz, T. Sauncy, and T. Dallas \\ Department of Physics, Texas Tech University, Lubbock, Texas 79409 \\ S. Massie* \\ Department of Physics, Virginia Polytechnic Institute and State University, Blacksburg, Virginia 24061
}

(Received 15 August 1994)

\begin{abstract}
We report cryogenic high-pressure measurements of a defect-related emission at $1.25 \mathrm{eV}$ in silicon-doped GaAs. The pressure measurements prove that the $1.25-\mathrm{eV}$ photon energy is relative to the conduction band, implying a deep defect level $0.30 \mathrm{eV}$ above the valence band and an electron-capture process from the conduction band into the defect. The defect level moves up in the band gap at a rate of $23 \pm 3 \mathrm{meV} / \mathrm{GPa}$. These results are consistent with a vacancy-related defect level, possibly stemming from a gallium-vacancy-siliconat-gallium (second-nearest-neighbor) defect complex.
\end{abstract}

Point defects occurring in semiconductors have been widely studied due to their technological relevance and because they pose numerous fundamental questions. ${ }^{1}$ Common to both issues is the position of defect-related levels introduced into the energy gap. In heavily doped semiconductors, the possibility that defect complexes that consist of native defects and substitutional dopant impurities exist in significant concentrations must be recognized. ${ }^{2}$ Identifying the microscopic defect origin of particular levels present in a semiconductor is an extremely challenging task. However, the study of plausible identifications of such defect signatures is important because any verifications facilitate examination and control of particular defect structures. We present one such study in this paper.

The experimental techniques used to explore these problems are diverse. Two important and related techniques which have been used fruitfully to investigate defects are spectroscopy under uniaxial stress ${ }^{3,4}$ and under hydrostatic pressure. ${ }^{5-9}$ Uniaxial stress measurements of defect-related states in GaAs are best used to break level degeneracies by lowering the symmetry of the defect, thereby splitting degenerate levels. The nain advantage of hydrostatic pressure as a means of perturbing a solid is that it isotropically decreases volume (i.e., interatomic spacing) in a bulk material. Shifts in energies are then due solely to the volume deformation induced by the pressure. In this paper we report on our use of hydrostatic pressure, in combination with low-temperature photoluminescence, to examine defect-related emission observed at $1.25 \mathrm{eV}$ in heavily silicon-doped GaAs. ${ }^{10-12}$ The objectives were to determine the exact position of the level in the band gap (i.e., emission from the defect level to the valence band or from the conduction band to the defect level), and to provide information about the microscopic origin of this level.

The silicon-doped GaAs was grown via molecular-beam epitaxy (MBE) at $580^{\circ} \mathrm{C}$. The GaAs:Si layer was grown on an undoped GaAs buffer layer that had been deposited on pure liquid-encapsulated Czochralski GaAs substrates. Growth-layer thicknesses ranged from 2000 to $6500 \AA$. Silicon concentrations ranged from $3.9 \times 10^{17}$ to $8.6 \times 10^{18}$ $\mathrm{cm}^{-3}$, which we determined by Hall measurements and con- firmed with Raman measurements of the phonon-plasmon energy. ${ }^{13}$ For the pressure studies, the GaAs:Si samples were backpolished to a total thickness of $\approx 30 \mu \mathrm{m}$. Samples were then cleaved into $\approx 100$ - $\mu \mathrm{m}$ squares and loaded into a miniature diamond-anvil cell. A 4:1 mixture of methanol and ethanol was used as a pressure-transmitting medium, and ruby was used to determine the pressure. ${ }^{14}$ All pressure changes were made at room temperature because previous cryogenic pressure studies indicate that a large amount of nonhydrostatic stress is introduced when the pressure is adjusted at low temperature. ${ }^{15}$ The cell was loaded into a dovetail cryostat with optical access and immersed in liquid nitrogen for the photoluminescence studies. All pressures reported were measured at $77 \mathrm{~K}$. Photoluminescence spectra were generated with a 514.5-nm argon-ion laser light with the intensity at the sample maintained below $10 \mathrm{~mW}$.

Photoluminescence spectra of three heavily silicon-doped gallium-arsenide samples are shown in Fig. 1. As the silicon donor concentration increases, the spectra rapidly broaden and shift upward in energy. ${ }^{16}$ This band-filling effect is com-

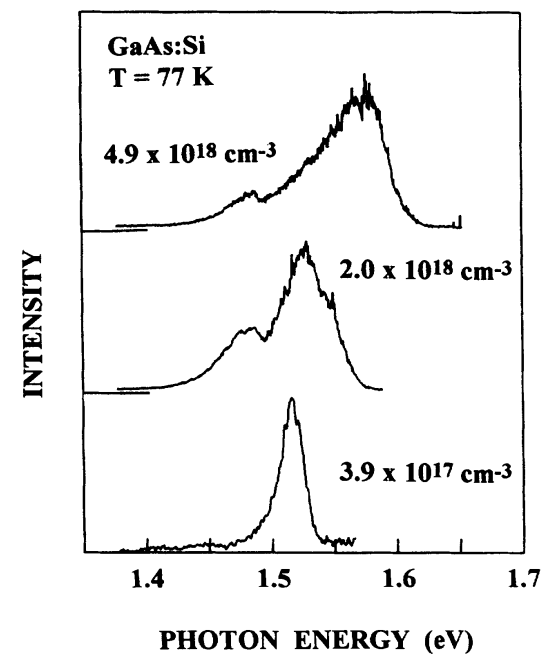

FIG. 1. Photoluminescence spectra of heavily silicon-doped MBE GaAs showing the band-gap emission at zero pressure. 


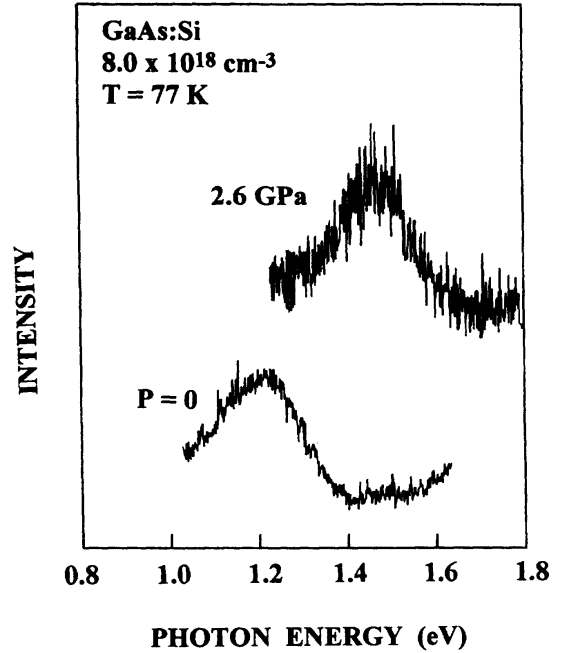

FIG. 2. Defect-related emission of very heavily silicon-doped GaAs. Spectra are shown at ambient pressure and at 2.6 GPa.

monly called the Burnstein-Moss shift, ${ }^{17}$ and our observations are consistent with what has been previously observed in heavily doped, MBE-grown GaAs. ${ }^{18}$ We also observed no band-gap-related emission in the most heavily doped sample studied $\left(8.0 \times 10^{18} \mathrm{~cm}^{-3}\right)$.

For the two highest dopant concentrations studied, $4.9 \times 10^{18}$ and $8.0 \times 10^{18} \mathrm{~cm}^{-3}$, we also observe the emergence of a very broad (200-meV) emission near $1.25 \mathrm{eV}$, previously attributed to a complex that consists of a donor and a gallium vacancy. ${ }^{10}$ This emission was not observed in samples with lower silicon concentrations, and therefore is related to the large defect concentration. In Fig. 2 we show the $77-\mathrm{K}$ spectrum of this band for the $n=8.0 \times 10^{18} \mathrm{~cm}^{-3}$ sample, for which the emission was more intense. Spectra are shown at ambient pressure and at $2.6 \mathrm{GPa}$. The linewidth remained approximately constant at $200 \mathrm{meV}$ over the entire pressure range examined (to $5.2 \mathrm{GPa}$ ), which provides evidence that the origin of the luminescence is the same at each pressure.

In Fig. 3 the peak photon energy of the emission shown in Fig. 2 vs pressure up to $5.2 \mathrm{GPa}$ is plotted. Below $4 \mathrm{GPa}$ the emission shifts approximately linearly with pressure according to

$\hbar \omega=(1.246 \pm 0.005 \mathrm{eV})+(0.084 \pm 0.003 \mathrm{eV} / \mathrm{GPa}) P$

Above $4 \mathrm{GPa}$ we observe that the emission photon energy diminishes with pressure. The approximate crossover pressure at which the two pressure behaviors (increasing below $P_{c}$ and decreasing above $P_{c}$ ) intersect is $P_{c}=3.9 \mathrm{GPa}$, which is the pressure at which the direct-indirect crossover occurs in pure GaAs. ${ }^{19}$

The pressure data summarized in Fig. 3 afford us a detailed picture of the defect level seen in this heavily doped, highly disordered GaAs. First and foremost, we conclude that the defect luminescence is due to the recombination of an electron from the conduction band with the ionized defect. That is, $1.25 \mathrm{eV}$ is the depth of the defect level relative to the conduction band. Such a conclusion would not have been possible without the high-pressure data. The recombi-

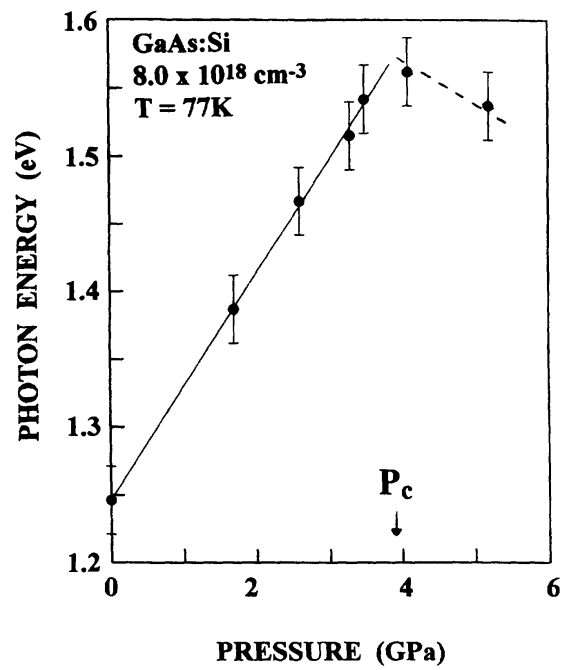

FIG. 3. Peak emission energy (from Gaussian fits to spectra) versus pressure. The two distinct slopes cross near $3.9 \mathrm{GPa}$, indicating that the emission is from conduction-band states into the defect level, as shown in Fig. 4.

nation process is shown pictorially in Fig. 4 for representative pressures below and above $P_{c}$.

Given the above deduction, we may now estimate the energy of the defect level from the valence band. This is complicated by the fact that the band gap of our heavily doped GaAs is not known exactly. Thus we cannot say that the conduction-band electrons come from the bottom of that

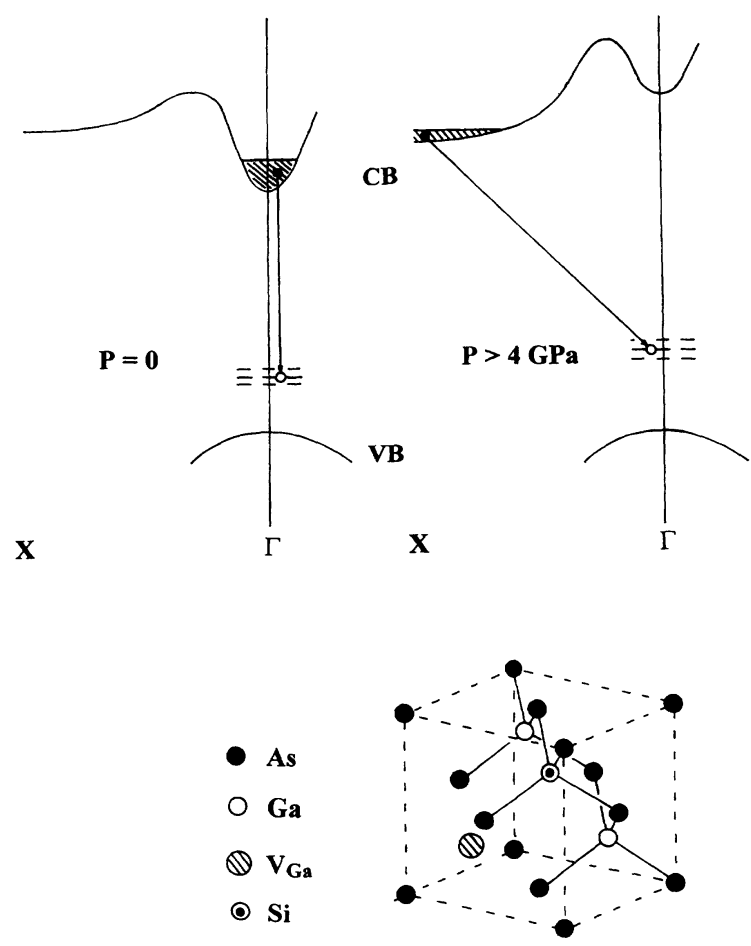

FIG. 4 . The emission process implied by Fig. 3 at ambient pressure and above $P_{c}$ (top). The plausible defect structure (bottom) responsible for the emission band studied. 
band for each pressure. It is much more likely that the electrons come from states throughout the band-filled energy range, consistent with the Burstein-Moss effect. This helps explain the large linewidth observed for the defect-related emission, which is a convolution of the filled conductionband states and the defect level. Our spectra imply a defect level spanning a $0.10-\mathrm{eV}$ energy range. If we now use the central energy of the uppermost spectrum in Fig. 1 (most heavily doped) as the average energy of conduction-band electrons, we estimate that the defect level being probed is $E_{D}=0.30 \mathrm{eV}$ above the valence band. This value is supported by the data above $P_{c}$, as will be discussed shortly.

Turning our attention to the pressure coefficient of the $1.25-\mathrm{eV}$ band, we notice that $84 \pm 3 \mathrm{meV} / \mathrm{GPa}$ is substantially smaller than the rate at which the direct band gap of pure GaAs increases [107.3 $\pm 0.5 \mathrm{meV} / \mathrm{GPa}$ (Ref. 19)]. However, the relative pressure shift

$$
d(\ln \hbar \omega) / d P_{P=0}=0.067 \pm 0.002 \mathrm{GPa}^{-1}
$$

is very close to the bulk GaAs value of $0.0708 \pm 0.0004$ $\mathrm{GPa}^{-1}$. Evidently, the defect level shifts relative to both the valence and conduction bands. The rate relative to either band edge is in approximate proportion to its separation from that edge. Shallow-donor levels are known to shift rigidly with the conduction band and shallow-acceptor levels shift with the valence band. ${ }^{19,20}$ The observed shift of the $1.25-\mathrm{eV}$ luminescence relative to both bands is consistent with the deep character of the related level.

The above pressure coefficient implies that the defect level shifts at a rate of approximately $d E_{D} / d P=23 \pm 3$ $\mathrm{meV} / \mathrm{GPa}$ (i.e., it moves higher in the band gap with pressure). Since the $X$ minimum moves closer in energy to the valence band, at a rate of $d E_{X} / d P=-13.4 \pm 0.4$ $\mathrm{meV} / \mathrm{GPa},{ }^{19}$ the defect level and the $X$ valley should approach each other at a rate of $-36 \pm 3 \mathrm{meV} / \mathrm{GPa}$ (the energy difference between the defect level and the $X$ conductionband minimum decreases with increasing pressure). If this implied pressure coefficient is used to extrapolate to $P=0$ from the crossover in our data, then the separation between the $X$ point and the defect level is estimated to be $1.70 \mathrm{eV}$. The dashed line in Fig. 3 shows the pressure behavior of the defect emission implied by this analysis. Summing this with the $E_{D}=0.30 \mathrm{eV}$ energy of the defect above the valence band, which was implied by the low-pressure behavior, we arrive at the accepted valence-band $(\Gamma)$-conduction-band $(X)$ indirect band gap of GaAs $\left[E_{X}=2.010 \mathrm{eV}\right.$ (Ref. 19)]. The fact that our numbers sum to something slightly different than the $\Gamma-X$ splitting is acceptable since, above $P_{c}$, we would expect some band filling of the states which correspond to the $X$ point in bulk material (shown in Fig. 4). Furthermore, the exact values of the two data points above $P_{c}$ should not be overemphasized-they serve primarily to determine the nature of the emission process. Taking these arguments into consideration, we feel the consistency of the pressure data is excellent.

One proposed origin for the defect-related emission studied here is a $\mathrm{V}_{\mathrm{Ga}}-\mathrm{Si}_{\mathrm{Ga}}$ (second-nearest neighbor, Fig. 4) pair complex. ${ }^{12}$ The presence of a high concentration of these defects in our samples is reasonable. MBE growth allows for vacancy formation and the gallium vacancy would be an acceptor, therefore making it electrostatically favorable to form a complex with the $\mathrm{Si}_{\mathrm{Ga}}$ donor. This would lead to partial compensation of the donors at high silicon concentrations. An alternative suggestion, that the $1.25-\mathrm{eV}$ emission arises from a $\mathrm{Cu}_{\mathrm{Ga}}-\mathrm{Si}_{\mathrm{Ga}}$ defect, ${ }^{12}$ is unlikely for our samples because copper was not introduced at any point in the preparation or measurement process. McQuaid et al. ${ }^{2}$ have recently observed infrared-absorption modes that they suggest arise from $\mathrm{V}_{\mathrm{Ga}}-\mathrm{Si}_{\mathrm{Ga}}$ complexes in heavily $\mathrm{Si}$-doped $\mathrm{GaAs}$, thus supporting our analysis that the $1.25-\mathrm{eV}$ emission stems from these defects. We note, however, that our results are consistent with a vacancy-related defect level. It is therefore important to point out that other defects involving vacancies, such as $\mathrm{V}_{\mathrm{As}}-\mathrm{Si}_{\mathrm{Ga}}$, which may also be present in sufficient concentrations, could be responsible for the emission we study. With this proviso, we shall proceed to consider the $\mathrm{V}_{\mathrm{Ga}}-\mathrm{Si}_{\mathrm{Ga}}$ complex as the most plausible defect microstructure.

The energy levels of the isolated vacancy have been calculated. $^{21}$ For the $\mathrm{V}_{\mathrm{Ga}}$ point defect, levels occur in the valence band and in the conduction band, but not in the gap. When in close proximity with the $\mathrm{Si}_{\mathrm{Ga}}$ donor, the vacancyrelated energy levels should shift down in energy due to the additional Coulombic binding. This simple picture will be complicated by local distortions when the defects are brought together to form a complex. Nonetheless, pressure will diminish the total volume of the defect and have the biggest effect on the vacancy. If we assume that the pressure compresses the vacancy with little effect on the bond lengths of the second-nearest neighbors, as in Ref. 22, the volume of $\mathrm{V}_{\mathrm{Ga}}$ will decrease at a rate of about $1.3 \AA^{3} / \mathrm{GPa}$, or

$$
\frac{1}{V_{0}} \frac{\Delta V}{\Delta P} \approx 0.086 \mathrm{GPa}^{-1}
$$

Presumably, we observe the $(+, 0)$ electron-capture process, ${ }^{23}$ in which the neutral state is the $\mathrm{Si}_{\mathrm{Ga}}-\mathrm{V}_{\mathrm{Ga}}$ defect (donor-acceptor, respectively). Samara, Vook, and Gibbons ${ }^{8}$ have studied the breathing-mode relaxation of the EL 2 defect in $\mathrm{GaAs}$, and find an implied volume deformation of 3.7 $\pm 3.0 \AA^{3}$ for electron capture. Their results support an arsenic antisite as the probable cause of the EL 2 level. They prescribe a method for determining the volume deformation from defect-level pressure shifts. In the case of the $\mathrm{Si}_{\mathrm{Ga}}-\mathrm{V}_{\mathrm{Ga}}$ defect, we are not dealing with a simple breathing-type deformation: the low symmetry introduced by the contraction around the silicon and the expansion around the vacancy is complicated. Nevertheless, using our pressure shift of $d E_{D} / d P=23 \pm 3 \mathrm{meV} / \mathrm{GPa}$ for the defect relative to the valence band, and using the absolute shift of the valence band under pressure of $d E_{v} / d P=6 \pm 1 \mathrm{meV} / \mathrm{GPa}$ used by Ref. 8, we arrive at a charge-capture volume deformation of $\Delta V_{c} \approx+2.7 \pm 0.6 \AA^{3}$. The sign and magnitude of this result are both consistent with what was found in Ref. 8, i.e., an expansion of the defect upon electron capture. Our higher precision is a consequence of the relative ease of the photoluminescence measurements and the greater range of pressure that can be accessed with diamond-anvil cell techniques, when compared with deep-level transient spectroscopy (DLTS) experiments performed under pressure. 
Our results support the DLTS analysis. Assuming that the arsenic antisite is the correct microscopic model for $E L 2$, our implied deformation is slightly smaller since (primarily) the vacancy of our defect complex must accommodate the extra electron and the total available volume is larger than for the arsenic antisite. The relevant upper energy levels of the defect will tend to have mixed bonding and antibonding character. The smaller ionized $(+)$ level will have enhanced bonding character, providing a lowering of the level into the gap. Evidently, pressure enhances the antibonding nature of this state, producing the positive pressure coefficient of the defect level involved in the emission process. Furthermore, a Franck-Condon-type shift is necessarily implied by the volume deformations associated with our $(+, 0)$ capture process, as in Refs. 20 and 9. We may conclude from this analysis that the pressures applied here result in volume deformations that are significant and in excess of $\Delta V_{c}$ due to the chargecapture process. A pressure shift of the defect level is therefore expected.

In conclusion, our experiment proves that the $1.25-\mathrm{eV}$ photoluminescence comes from a conduction-band to defect radiative recombination. The defect level is approximately $0.30 \mathrm{eV}$ above the valence band, a result that is consistent with the low-pressure (below the direct-indirect crossover near $4 \mathrm{GPa}$ ) and high-pressure data. The pressure coefficients are consistent with a deep defect level which shifts relative to both the valence and conduction bands. Our pressure data are consistent with a vacancy-related defect complex and support a previous suggestion that the $1.25-\mathrm{eV}$ emission band is due to electron-capture luminescence by the $\mathrm{V}_{\mathrm{Ga}}-\mathrm{Si}_{\mathrm{Ga}}$ defect.

The authors wish to thank F. G. Anderson for a critical reading of this manuscript and helpful discussions.
*Present address: Quantum Epitaxial Design, 119 Technology Drive, Bethlehem, PA 18015.

${ }^{1}$ See Proceedings of the 17th International Conference of Defects in Semiconductors, edited by $\mathbf{H}$. Heinrich and W. Jantsch [Mater. Sci. Forum 143-147 (1994)].

${ }^{2}$ S. A. McQuaid, R. C. Newman, M. Missous, and S. O'Hagan, Appl. Phys. Lett 51, 3008 (1992).

${ }^{3}$ M. K. Nissen, A. Villemaire, and M. L. W. Thewalt, Phys. Rev. Lett. 67, 112 (1991).

${ }^{4}$ S. Yang and C. D. Lamp, Phys. Rev. B 49, 1690 (1994).

${ }^{5}$ M. F. Li, P. Y. Yu, E. R. Weber, and W. Hansen, Phys. Rev. B 36, 4531 (1987).

${ }^{6}$ W. Shan, P. Y. Yu, M. F. Li, W. L. Hansen, and E. Bauser, Phys. Rev. B 40, 7831 (1989).

${ }^{7}$ M. F. Li, P. Y. Yu, W. Shan, W. Hansen, and E. R. Weber, Appl. Phys. Lett. 54, 1344 (1989).

${ }^{8}$ G. A. Samara, D. W. Vook, and J. F. Gibbons, Phys. Rev. Lett. 68, 1582 (1992).

${ }^{9}$ J. A. Wolk, W. Walukiewicz, M. L. W. Thewalt, and E. E. Haller, Phys. Rev. Lett. 68, 3619 (1992).

${ }^{10}$ J. I. Pankove, Optical Processes in Semiconductors (Dover, New York, 1971), p. 140.
${ }^{11}$ E. V. K. Rao and N. Duhamel, J. Appl. Phys. 49, 3458 (1978).

${ }^{12}$ J. van de Ven, W. J. A. M. Hartmann, and L. J. Giling, J. Appl. Phys. 60, 3735 (1986).

${ }^{13}$ A. Mooradian and G. Wright, Phys. Rev. Lett. 16, 999 (1966).

${ }^{14}$ G. J. Piermarini, S. Block, J. D. Barnett, and R. A. Forman, J. Appl. Phys. 46, 2779 (1975).

${ }^{15}$ K. Reimann and K. Syassen, Phys. Rev. B 39, 11113 (1989).

${ }^{16}$ The weaker feature near $1.48 \mathrm{eV}$ stems from the buffer layer.

${ }^{17}$ E. Burstein, Phys. Rev. 93, 632 (1954); T. S. Moss, Proc. Phys. Soc. London Sect. B 76, 775 (1954).

${ }^{18}$ Jiang De-Sheng, Y. Makita, K. Ploog, and H. J. Queisser, J. Appl. Phys. 53, 999 (1982).

${ }^{19}$ D. J. Wolford and J. A. Bradley, Solid State Commun. 53, 1069 (1985).

${ }^{20}$ A. Zylbersztejn, R. H. Wallis, and J. M. Besson, Appl. Phys. Lett. 32, 764 (1978).

${ }^{21}$ C. W. Myles and O. F. Sankey, Phys. Rev. B 29, 6810 (1984).

${ }^{22}$ T. Sauncy, M. Holtz, and R. Zallen, Phys. Rev. B 50, 10702 (1994).

${ }^{23}$ The $(+, 0)$ process could also be the $(++,+)$ electron capture; we mention only the return to the ground state in the text. 